\title{
EUCLIDEAN SUBRINGS OF GLOBAL FIELDS ${ }^{1}$
}

\author{
BY CLIFFORD S. QUEEN \\ Communicated by Hyman Bass, October 20, 1972
}

1. Introduction. The purpose of this note is to announce some results regarding the existence of euclidean subrings of global fields.

We first state the problem and give its history. Let $F$ be a global field. So $F$ is a finite extension of the rational numbers $Q$ or $F$ is a function field of one variable over a finite field $k$, where $k$ is algebraically closed in $F$. Let $S$ be a finite nonempty set of prime divisors of $F$ such that $S$ includes all infinite (i.e., archimedean) prime divisors. If $P$ is a finite (i.e., nonarchimedean) prime divisor we denote by $O_{P}$ its valuation ring in $F$. Now, given a finite set $S$ of the above type, we get a ring

$$
O_{S}=\bigcap_{P \notin S} O_{P}
$$

where $P$ ranges over all prime divisors of $F$. We note in particular that if $F$ is a number field and $S$ the set of infinite prime divisors of $F$ then $O_{S}$ is just the ring of $F$-integers.

It is easy to see that there always exists a finite set $S$ satisfying the above hypothesis such that $O_{S}$ is a unique factorization domain. Hence it seems natural to ask the following two questions:

I. Does there always exist an $S$ such that $O_{S}$ is a euclidean ring?

II. Can one find an algorithm on $O_{S}$ for suitably chosen $S$ which is related in some way to the arithmetic of the field $F$ ?

The history of the above two questions is as follows: In a series of articles [1]-[4] Armitage discussed I and II for function fields over arbitrary ground fields. He insisted on a choice of algorithm related to the norm from $F$ to a rational subfield. He showed that if the ground field of $F$ is infinite, then an algorithm of his spacial type was possible if and only if the genus of $F$ is zero. He also discussed the case when the ground field of $F$ is finite, but again the only situation in which he gave a positive answer to I was when $F$ is of genus zero. In [6], Samuel also discussed I for function fields $F$ with arbitrary fields of constants, but here also he did not get above genus zero. Finally, in [5], M. Madan and the present author showed that the answer to both I and II is yes for function fields of genus one over finite fields. The method in [5] was to specifically construct an $S$ and an algorithm on $O_{S}$ for given $F$.

AMS (MOS) subject classifications (1970). Primary 12A05; Secondary 12A05.

Key words and phrases. Euclid's algorithm, adeles, global fields.

${ }^{1}$ ADDED IN PROOF. After this announcement went to press, the author discovered that Theorem 2 was proved by O. T. O'Meara in On the finite generation of linear groups over Hasse domains, J. Reine Angew. Math. 217 (1965), 79-108. MR 31 \# 3513. 
In the next section we indicate a proof that the answer to both I and II is yes for arbitrary global fields $F$. Full details of the proof and applications of the results will appear elsewhere.

2. Results. Let $F$ be a global field. If $P$ is a finite prime divisor of $F$ we denote by $N(P)$ the absolute norm of $P$ and we associate with each such $P$ a normalized valuation ||$_{P}$ as follows: $|O|_{P}=0$ and if $x \in F-\{0\}=F^{*}$, then $|x|_{P}=N(P)^{-n}$, where $P^{n}$ is the power to which $P$ appears in the principal divisor $(x)$ determined by $x$ in $F$. Now if $P$ is an infinite prime divisor then $P$ corresponds to an embedding $\sigma_{P}$ of $F$ into the complex numbers and we determine a normalized valuation ||$_{P}$ associated to $P$ in the following way: If $\sigma_{P}(F)$ is a subfield of the real numbers then $|x|_{P}$ $=\left|\sigma_{P}(x)\right|$ for all $x \in F$, where || is the ordinary real absolute value. Finally if $\sigma_{P}(F)$ is not a subfield of the reals, we set $|x|_{P}=\left|\sigma_{P}(x)\right|^{2}$ for all $x \in F$, where || is the usual complex absolute value. Hence letting $P$ range over all prime divisors of $F$, we have the well-known formula

$$
\prod_{P}|x|_{P}=1
$$

for all $x \in F^{*}$.

If $P$ is a prime divisor of $F$ we denote by $F_{P}$ the completion of $F$ with respect to the valuation ||$_{P}$. These fields $F_{P}$ are all locally compact and if $P$ is finite we denote by $R_{P}$ the maximal compact subring of $F_{P}$. We call the restricted topological product of the $F_{P}$ with respect to the $R_{P}$ the ring of adeles of $F$ and denote it by $F_{A}$. We further identify $F$ with its diagonal embedding in $F_{A}$.

Now if $F$ is a number field we denote by $S_{\infty}$ the set of infinite prime divisors of $F$ and if $F$ is a function field over a finite field we fix a prime divisor $P_{\infty}$ of $F$ and set $S_{\infty}=\left\{P_{\infty}\right\}$. Next if $x \in F^{*}$ we set

$$
V(x)=\left\{\left.\zeta \in F_{A}|| \zeta_{P}\right|_{P}<|x|_{P} \text { for } P \in S_{\infty} \text { and }\left|\zeta_{P}\right|_{P} \leqq|x|_{P} \text { for } P \notin S_{\infty}\right\} .
$$

THEOREM 1.

$$
F_{A}=\bigcup_{x \in F^{*}}(V(x)+F) .
$$

INDICATION OF PROOF. If $F$ is a function field and $k$ its exact field of constants we use the Riemann-Roch theorem to choose $t \in F$ such that $F / k(t)$ is a separable extension and $|t|_{P_{\infty}}>1$, with $|t|_{P} \leqq 1$ for all $P \neq P_{\infty}$. If $F$ is a number field we let $H$ denote the field of real numbers and otherwise $H$ will denote $k\left(\left(t^{-1}\right)\right)$, where $k\left(\left(t^{-1}\right)\right)$ is the quotient field of the ring of formal power series in $t^{-1}$ over $k$. Next we set $F_{\infty}=F \otimes_{L} H$, where $L=Q$ if $F$ is a number field and $L=k(t)$ otherwise. Viewing $F_{\infty}$ as a topological algebra over $H$ we identify it with the subalgebra of $F_{A}$, $\prod_{P \in S_{\infty}} F_{P}$. 
Setting $X=F_{\infty} \times \prod_{P \notin S_{\infty}} R_{P}$, we observe that $F_{A}=X+F$ (see [7]). Let $\left\{\omega_{1}, \ldots, \omega_{n}\right\}$ be an integral basis of $F$ over $L$ with respect to $\Gamma$, where $\Gamma$ is the ring of rational integers if $F$ is a number field and otherwise $\Gamma=k[t]$. Finally we show that if $\zeta \in X$, then there exist $q, p_{1}, \ldots, p_{n} \in \Gamma$ such that $q \neq 0$ and $q \zeta-\left(p_{1} \omega_{1}+\cdots+p_{n} \omega_{n}\right)$ has the property that

and

$$
\left|\left(q \zeta-\left(p_{1} \omega_{1}+\cdots+p_{n} \omega_{n}\right)\right)_{P}\right|_{P}<1 \text { for } P \in S_{\infty}
$$

$$
\left|\left(q \zeta-\left(p_{1} \omega_{1}+\cdots+p_{n} \omega_{n}\right)\right)_{P}\right|_{P} \leqq 1 \quad \text { for } P \notin S_{\infty},
$$

i.e., $\zeta \in V\left(q^{-1}\right)+F$. Q.E.D.

Let $S$ be a finite set of prime divisors of $F$ such that $S \supseteq S_{\infty}$. We define a function $\varphi_{S}$ from $F$ to the nonnegative real numbers given by $\varphi_{S}(x)$ $=\prod_{P \in S}|x|_{P}$. We note that, in view of (1), $\varphi_{S}$ is integral valued when restricted to $O_{S}$. Further in the case when $F$ is a number field and $S=S_{\infty}$, then, for all $x \in F, \varphi_{S}(x)=\left|N_{F / Q}(x)\right|$. Also when $F$ is a function field, then for any choice of $S \supseteq S_{\infty}$, there exist $y \in F-k$ such that $O_{S}$ is the integral closure of $k[y]$ in $F$ and, for all $x \in F, \varphi_{S}(x)=\left|N_{F / k(y)}(x)\right|_{\infty}$, where ||$_{\infty}$ is the valuation associated to the pole divisor of $y$ in $k(y)$ and normalized as above.

THEOREM 2. There exists a finite set $S$ of prime divisors of $F$ such that $S \supseteq S_{\infty}$ and $O_{S}$ is euclidean with respect to the map $\varphi_{S}$.

INDICATION OF PROOF. By Theorem $1, F_{A}=\bigcup_{x \in F^{*}}(V(x)+F)$. Now by compactness of $F_{A} / F$ (see [7]) and the fact that $V(x)$ is open in $F_{A}$ for every $x \in F$, there exist $x_{1}, \ldots, x_{r} \in F^{*}$ such that

$$
F_{A}=\bigcup_{i=1}^{r}\left(V\left(x_{i}\right)+F\right) \text {. }
$$

Finally we show that if $S=\left\{P \mid P \in S_{\infty}\right.$ or there exist $i_{0}, 1 \leqq i_{0} \leqq r$ such that $\left.\left|x_{i_{0}}\right|_{P} \neq 1\right\}$, then $S$ is a finite set, $S \supseteq S_{\infty}$ and $O_{S}$ is euclidean with respect to $\varphi_{S}$.

\section{REFERENCES}

1. J. V. Armitage, Euclid's algorithm in certain algebraic function fields, Proc. London Math. Soc. (3) 7 (1957), 498-509. MR 20 \# 30.

2. - Corrigendum and Addendum: "Euclid's algorithm in algebraic function fields", J. London Math. Soc. 43 (1968), 171-172. MR 37 \#2727.

3. - On unique factorization in algebraic function fields, Illinois J. Math. 11 (1967), 280-283; Appendix, Illinois J. Math. 12 (1968), 5-6. MR 35 \# 172; MR 36 \# 5113.

4. $\ldots$, Euclid's algorithm in algebraic function fields. II, Acta Arith. 18 (1971), 337-348.

5. M. Madan and C. Queen, Euclidean function fields, J. Reine Angew. Math. (submitted).

6. P. Samuel, About Euclidean rings, J. Algebra 19 (1971), 282-301. MR 43 \# 6190.

7. A. Weil, Basic number theory, Die Grundlehren der math. Wissenschaften, Band 144, Springer-Verlag, New York, 1967. MR 38 \# 3244.

Department of Mathematics, Lehigh University, Bethlehem, Pennsylvania 18015 